\title{
Conditioned medium from human palatine tonsil mesenchymal stem cells attenuates acute graft-vs.-host disease in mice
}

\author{
KYUNG-AH CHO ${ }^{1}$, YU-HEE KIM ${ }^{1}$, MINHWA PARK ${ }^{1}$, HYE JI KIM ${ }^{1}$, SO-YOUN WOO ${ }^{1}$, \\ JOO-WON PARK ${ }^{2}$ and KYUNG-HA RYU ${ }^{3}$
}

Departments of ${ }^{1}$ Microbiology, ${ }^{2}$ Biochemistry and ${ }^{3}$ Pediatrics, College of Medicine,

Ewha Womans University, Seoul 07985, Republic of Korea

Received June 25, 2018; Accepted November 9, 2018

DOI: $10.3892 / \mathrm{mmr} .2018 .9659$

\begin{abstract}
Graft-vs.-host disease (GVHD) is a severe and potentially life-threatening complication of hematopoietic stem cell transplantation. Approximately $50 \%$ of patients exhibiting GVHD will not benefit from conventional steroid treatment. Although several second-line treatments are available for these patients, their prognoses remain poor due to the increased risk of infection, immunosuppression-mediated toxicity and incomplete GVHD remission, which occurs in the majority of cases. Mesenchymal stem cells (MSCs), a multipotent cell population, possess broad immunosuppressive activity and are a reportedly effective treatment of GVHD. However, the therapeutic effects of conditioned medium from MSCs on GVHD have not been demonstrated. In the present study, the efficacy of conditioned medium from human palatine tonsil-derived MSCs (T-MSC-CM) was validated against GVHD in mice. The suppressive function of T-MSC-CM on immune cell chemotaxis was confirmed in vitro. A systemic infusion of T-MSC-CM in mice with GVHD resulted in prolonged survival, rapid recovery from weight loss and reduced pathological damage in numerous GVHD-targeted organs. Furthermore, lymphocyte gene expression was significantly downregulated in GVHD mice administered T-MSC-CM. These results indicate that T-MSC-CM is a promising cellular agent to prevent or treat transplantation-associated complications such as GVHD.
\end{abstract}

\section{Introduction}

Allogeneic hematopoietic stem cell transplantation (HSCT) is an effective therapy for a number of hematological disorders.

Correspondence to: Professor Kyung-Ha Ryu, Department of Pediatrics, College of Medicine, Ewha Womans University, 1071 Anyangcheon-ro Yangcheon-gu, Seoul 07985, Republic of Korea

E-mail: ykh@ewha.ac.kr

Key words: allogeneic hematopoietic stem cell transplantation, palatine tonsil mesenchymal stem cell, graft-vs.-host disease, TSG-6, immune cell migration
Despite good progress in the prevention and treatment of complications that are often associated with transplantation, acute graft-vs.-host disease (aGVHD) occurs in 30-70\% of patients undergoing allogeneic HSCT and remains a leading cause of nonrelapse mortality $(1,2)$. This disease occurs when immune cells transplanted from a genetically non-identical donor recognize and are activated by alloantigens in HSCT recipients, resulting in organ damage, predominantly to the skin, gastrointestinal (GI) tract, and liver. Corticosteroids, which elicit a response rate of $50-80 \%$, are considered the first-line treatment for aGVHD $(2,3)$. However, patients who are unresponsive to this initial therapy exhibit only a $10-30 \%$ likelihood of long-term survival $(1,4)$. Although available, second-line pharmacological strategies are limited by their substantial impairment of the recipient's immune system and subsequent increases in opportunistic infections $(5,6)$. Therefore, the development of novel treatment strategies to improve the overall survival of HSCT recipients is of significant clinical relevance.

Mesenchymal stem cells (MSCs) are a type of multipotent adult stem cell that can be isolated from several tissues, including the bone marrow (BM), adipose tissue, and palatine tonsils. MSCs possess the capacity to suppress immunological responses, support hematopoiesis, and stimulate tissue repair $(7,8)$. Clinical applications of human MSCs for the prevention and treatment of GVHD are evolving rapidly. To this end, clinical studies have demonstrated the efficacy of systemic infusions of culture-expanded allogeneic human BM-MSCs for the treatment of patients with steroid-refractory aGVHD (9). In fact, allogeneic BM-MSC products have been used clinically in some countries as off-the-shelf treatments for steroid-resistant aGVHD (10). Previous evidence has suggested that MSCs inhibit immune cell functions primarily through the local secretion of soluble immune modulators and partially through cell-to-cell contact-dependent mechanisms. However, although MSCs are thought to be a less important source of immunogenic cells for transplantation, the half-life of infused MSCs and the risk of immune rejection following either their repeated administration or their use at high doses have not clearly been defined. Further, GVHD-affected organs may require more 'remote' and 'efficient' immunomodulatory effects following systemic infusion to minimize the loss of cells that directly adhere to tissues. 
Thus, the use of conditioned medium (CM) derived from MSCs (MSC-CM) could be a viable cellular approach to overcoming the limitations of the use of MSCs directly as a clinical treatment. Previously, we demonstrated that human palatine tonsil-derived MSCs (T-MSCs) abundantly secrete immunomodulatory proteins and that T-MSC-CM effectively attenuates inflammation both in vitro and in vivo (11-13).

In the current study, we examined the effects of T-MSC-CM on the prevention of GVHD in a mouse model of the disease. Survival, weight loss, pathological changes, and lymphocyte gene expression were evaluated to address the efficacy of T-MSC-CM as an alternative treatment for GVHD in patients undergoing HSCT.

\section{Materials and methods}

Animals. Female BALB/c and male C57BL/6 mice were purchased from OrientBio (Eumsung, Korea). All animals were maintained at $21-23^{\circ} \mathrm{C}$ with $51-54 \%$ humidity under pathogen-free conditions on a 12-h light/dark cycle with free access to food and water. All procedures were approved by the Animal Care and Use Committee of College of Medicine, Ewha Womans University (Seoul, Korea; approval no. ESM18-0403).

Preparation of $C M$. To generate MSC-CM, BM-MSCs, adipose tissue-derived MSCs (AT-MSCs), and T-MSCs (at passages 7-8) were cultured in low glucose Dulbecco's modified Eagle's medium (DMEM; Welgene, Daegu, Korea) in 100-mm tissue culture plates. The T-MSCs were obtained and maintained as previously reported (14). The T-MSCs were obtained and maintained as previously reported (14). The AT-MSCs were generously provided by RNL Bio (Seoul, Korea), and the BM-MSCs were purchased from the Severance Hospital Cell Therapy Center (Seoul, Korea). At $80 \%$ confluence, the cells were washed four times with PBS, and the medium was replaced with serum-free DMEM to generate CM. The medium was collected after $48 \mathrm{~h}$ of culture as previously reported $(15,16)$, centrifuged at 1,300 $\mathrm{rpm}$ for $5 \mathrm{~min}$, and passed through a $0.2-\mu \mathrm{m}$ filter. The CM was concentrated 20 -fold by centrifugal filtration by using centricone (molecular weight cut-off value of 3K; Amicon Ultra-15; EMD Millipore, Billerica, MA, USA) that provide highest yield in protein recovery. The concentrated $\mathrm{CM}$ was then frozen and stored at $-80^{\circ} \mathrm{C}$ for future use. As a negative control, the serum-free culture medium was processed in the same manner.

Western blotting. Equal amounts of CM from each MSC type (BM-MSCs, AT-MSCs, and T-MSCs) were loaded onto a polyacrylamide gel, separated by electrophoresis, transferred to polyvinylidene difluoride membranes, blocked, and incubated with primary antibodies overnight at $4^{\circ} \mathrm{C}$. The following primary antibodies were used: TSG-6 (1:200, diluted in 3\% BSA (Bovogen Biologicals, Pty, Ltd., East Keilor, Victoria, Australia) containing TBST; cat. no. sc-398307); and $\beta$-actin [1:3,000; diluted in 3\% BSA containing TBST; cat. no. sc-47778; both Santa Cruz Biotechnology, Inc., Dallas, TX, USA]. The membranes were washed 3 times for $10 \mathrm{~min}$ in TBST and incubated with anti-mouse (cat. no. BR170-6516; Bio-Rad Laboratories, Inc., Hercules, CA, USA) horseradish peroxidase-conjugated secondary antibodies (1:3,000; diluted in TBST) for $1 \mathrm{~h}$ at room temperature. Following incubation, membranes were washed 3 times for $10 \mathrm{~min}$ in TBST and developed using SuperSignal West Femto Maximum Sensitivity Substrate (Pierce; Thermo Fisher Scientific, Inc., Waltham, MA, USA). Images were obtained using ImageQuant LAS 4000 (GE Healthcare Life Sciences, Little Chalfont, UK). The pixel densities of the TSG-6 bands were divided by the pixel densities of the corresponding $\beta$-actin bands for the quantitation of protein levels using UN-SCAN-IT-gel 6.1 software (Silk Scientific, Inc., Orem, UT, USA).

ELISA. To quantify the amounts of TSG-6 secreted from BM-MSCs, AT-MSCs, and T-MSCs, CM was collected, and the levels of secreted TSG-6 were determined using a human TSG-6 ELISA kit, in accordance with the manufacturer's recommended protocol (cat. no. ELH-TSG-6; RayBiotech, Norcross, GA, USA).

In vitro chemotaxis assay. Spleen and draining lymph node (dLN) cells isolated from normal healthy male C57BL/6 mice were suspended in chemotaxis medium composed of RPMI-1640 (Welgene), 1\% fatty acid-free bovine serum albumin, $2 \mathrm{mM}$ glutamine, and $20 \mathrm{mM}$ HEPES. The cells ( $2 \times 10^{6}$ in $100 \mu \mathrm{l}$ chemotaxis medium) were placed in the upper chamber of 24-well Transwell plates ( $5 \mu \mathrm{M}$ pore size; Costar, Corning, NY, USA); $600 \mu 1$ chemotaxis medium lacking chemokines (control) or containing recombinant CCL2 (Peprotech, Rocky Hill, NJ, USA) at various concentrations $(50,100$, and $200 \mathrm{ng} / \mathrm{ml})$ were placed in the lower chamber. T-MSC-CM generated from $10^{6}$ cells or rhTSG-6 $(200 \mathrm{ng} / \mathrm{ml}$; Peprotech) was added to the lower chamber separately. The number of SP or dLN cells that migrated into the lower chamber after $4 \mathrm{~h}$ was determined by trypan blue staining.

Induction of GVHD. Female BALB/c recipient mice received busulfan (BU; $20 \mathrm{mg} / \mathrm{kg} /$ day) daily for 4 days, followed by cyclophosphamide (CY; $100 \mathrm{mg} / \mathrm{kg} /$ day) daily for 2 days via intraperitoneal injection. After 1 day of rest, a BM transfer (BMT) was performed on day 0 , as previously described $(17,18)$. For BM cells (BMCs) isolation, male C57BL/6 donor mice were killed by cervical dislocation and their limbs were removed. The BM was flushed from the medullary cavities of both the femurs and tibias and a single cell suspension was prepared. For the spleen cells (SPC), the spleen of male C57BL/6 donor mice was minced and dispersed into a single-cell suspension. After pelleting those cells, the erythrocytes were lysed using hypotonic buffer containing $0.75 \% \mathrm{NH} 4 \mathrm{Cl}$.

Female BALB/c recipient mice were injected with $1 \times 10^{7}$ BMCs combined with $1.5 \times 10^{7}$ SPCs in a total volume of $200 \mu \mathrm{l}$ via lateral tail vein injection (GVHD group). Mice transplanted with BMCs alone that did not induce GVHD served as the healthy control group as we previously established (18-20). Mice transplanted with BMCs and SPCs suspended in T-MSC-CM generated from $10^{6}$ cells were defined as the GVHD-T-MSC-CM group. The GVHD-T-MSC-CM group was injected with T-MSC-CM (from $10^{6}$ cells, $200 \mu \mathrm{l}$ ) once more after 3 days via the tail veil. The expected concentration of TSG-6 in the T-MSC-CM injection was $300 \mathrm{ng}$ per mouse. Mice transplanted with BMCs, SPCs, and rhTSG-6 
(1 ug/mouse) simultaneously via the tail vein were defined as the GVHD-rhTSG-6 group. We used eighteen female recipient mice and eighteen male donor mice per group. Thus, total number of animals used in the study is one hundred forty four.

During the experimental periods, humane endpoints was determined as the time when the animal lose weight over $20 \%$ from the starting weight. In that case, mice showed poor mobility and we immediately sacrificed.

Assessment of GVHD. Recipient mice were examined daily for 3 weeks; survival and weight loss were recorded. For clinical scoring, mice were sacrificed by cervical dislocation on days 7 and 21 following GVHD induction. Tissue samples from the liver, small intestine, large intestine, and skin were collected and fixed in $4 \%$ paraformaldehyde, and embedded in paraffin. After sectioning, the tissue sections were stained with hematoxylin and eosin and analyzed to confirm the presence of GVHD. To assess the severity of GVHD in mouse organs, seven parameters for skin (necrotic keratinocytes, lymphoid infiltration of the dermis, lymphocyte exocytosis, vascular degeneration of the epidermal-dermal junction, intraepithelial lymphoid infiltration, deficient Langerhans cells, and edema of the intercellular space), small intestine (villous blunting, crypt regeneration, crypt epithelial cell apoptosis, crypt loss, intraintestinal obstruction by cell debris, inflammatory cell infiltration of the lamina propria, and mucosal ulceration), and large intestine (crypt regeneration, crypt epithelial cell apoptosis, crypt loss, liquefaction of superficial epithelial cells, degeneration of superficial epithelial cells, inflammatory cell infiltration of the lamina propria, and mucosal ulceration) were scored, whereas 10 parameters (portal tract expansion by inflammatory cell infiltrates, lymphocyte infiltration of bile ducts, bile duct epithelial cell apoptosis, bile duct epithelial cell sloughing, vascular endothelialitis, parenchymal apoptosis, parenchymal microabscesses, parenchymal mitotic figures, hepatocellular cholestasis, and hepatocellular steatosis) were scored for the liver. The scoring of each parameter was as follows: 0 , normal; 0.5 , focal and rare; 1 , focal and mild; 2, diffuse and mild; 3 , diffuse and moderate; and 4, diffuse and severe. The scores were added to achieve a total score for each organ; therefore, the maximum score was 28 for the skin, small intestine, and large intestine, and 40 for the liver.

Reverse transcription-quantitative polymerase chain reaction. To confirm the expression of CD4 and CD19 in the skin, liver, small intestine, and large intestine, total RNA was extracted from organs harvested on day 21 after GVHD induction. Total RNA $(1 \mu \mathrm{g})$ was transcribed into complementary DNA using a reverse transcription reagent (ELPIS-Biotech Inc., Daejeon, Korea), according to the manufacturer's instructions. Amplification was performed in duplicate by 40 cycles of $15 \mathrm{sec}$ denaturation step at $95^{\circ} \mathrm{C}$ and a $1 \mathrm{~min}$ amplification and signal acquisition step at $60^{\circ} \mathrm{C}$ using StepOnePlus Real-Time PCR System (Applied Biosystems; Thermo Fisher Scientific, Inc.) using SYBR-Green (Kapa Biosystems, Inc., Wilmington, MA, USA). All gene expression values were normalized to the expression of the GAPDH reference gene using the following primers: mouse CD4 (115 bp) forward, 5'-TCCTAGCTG TCACTCAAGGGA-3' and reverse, 5'-TCAGAGAACTTC CAGGTGAAGA-3'; mouse CD19 (164 bp) forward, 5'-GGA
GGCAATGTTGTGCTGC-3' and reverse, 5'-ACA ATC ACTAGCAAGATGCCC-3'; and mouse GAPDH (173 bp) forward, 5'-GGTAAAGTGGATATTGTTGCCATCAATG-3' and reverse, 5'-GGAGGGATCTCGCTCCTGGAAGATGGT G-3'. The relative fold expression and changes were calculated $2^{-\Delta \Delta C t}$ method (21).

Statistical analysis. Data are presented as the mean \pm standard error of the mean. Statistical significance was determined by two-way analysis of variance (ANOVA) in conjunction with Dunnett's post hoc test over non-treated group for chemotaxis assay. Two-way ANOVA in conjunction with Dunnett's post hoc test over GVHD group were used for weight loss and total clinical scoring. One-way ANOVA in conjunction with Sidak's post-hoc test were used in quantitative real-time PCR. Survival curves were plotted using Kaplan-Meier estimates. All analysis was performed using GraphPad Prism, version 7 software (GraphPad Software, Inc., La Jolla, CA, USA). For all analyses, $\mathrm{P}<0.05$ was considered to indicate a statistically significant difference..

\section{Results}

T-MSCs secrete TSG-6 and effectively inhibit chemotaxis diverse cell populations including immune cells. The recruitment of donor immune cells, including $\mathrm{T}$ and $\mathrm{B}$ cells, into recipient target organs is critical for the maximal induction of GVHD. Based on our previous findings that T-MSCs abundantly secrete immunomodulatory cytokines, we sought to determine whether T-MSCs secrete proteins that regulate immune cell migration. TSG-6 was originally considered a potent inhibitor of neutrophil extravasation via the disruption of CXCL8 activity (22), but TSG-6 also diminishes the activity of various chemokines, including CXCL4, CXCL12, CCL2, CCL5, CCL7, CCL19, CCL21, and CCL27, by direct binding (23). Thus, we tested whether T-MSCs produce TSG-6 endogenously and whether either T-MSC-CM or TSG-6 inhibits the migration of a heterogeneous population of immune cells under chemotactic conditions. As shown in Fig. 1A, the secreted form of TSG-6 was abundantly generated by both T-MSCs and AT-MSCs without any preconditioning, but not by BM-MSCs, according to the results of the western blot analysis. Secreted TSG-6 was detected at high levels in T-MSCs, according to the ELISA results (Fig. 1B). Next, we investigated the effects of TSG- 6 on CCL2, a potent chemokine commonly produced by target organs in GVHD, using a Transwell assay. The concentration of TSG-6 in T-MSC-CM ( $10^{6}$ cell derived) is $136 \mathrm{ng}$, and the final concentration of TSG-6 in T-MSC-CM is expected as $217 \mathrm{ng} / \mathrm{ml}$ in the Transwell assay. We choose $10^{6}$ cells-derived T-MSC-CM as the concentration of TSG-6 in T-MSC-CM is most similar when we use $200 \mathrm{ng} / \mathrm{ml}$ of rhTSG-6. In this system, CCL2 upregulated the migration of SPCs and dLN cells in a dose-dependent manner. Under these conditions, we showed that T-MSC-CM and rhTSG-6 independently ablated the CCL2-induced migration of immune cells, which was significantly more pronounced in SPCs than in dLN cells (Fig. 1C and D). Given that the T cells used in this assay were not purified, the inhibitory effects of TSG-6 or TSG-6-containing T-MSC-CM may extend beyond specific cell types. 
A DMEM BM-MSC AT-MSC T-MSC
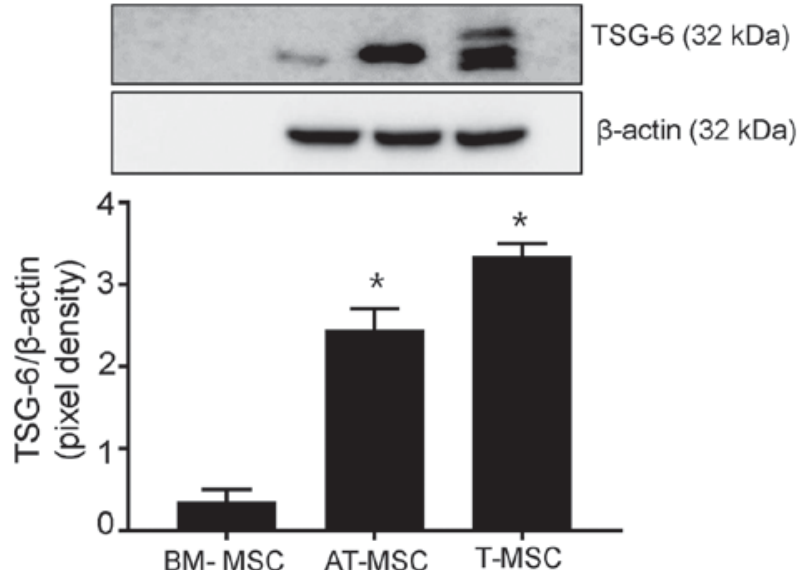

C

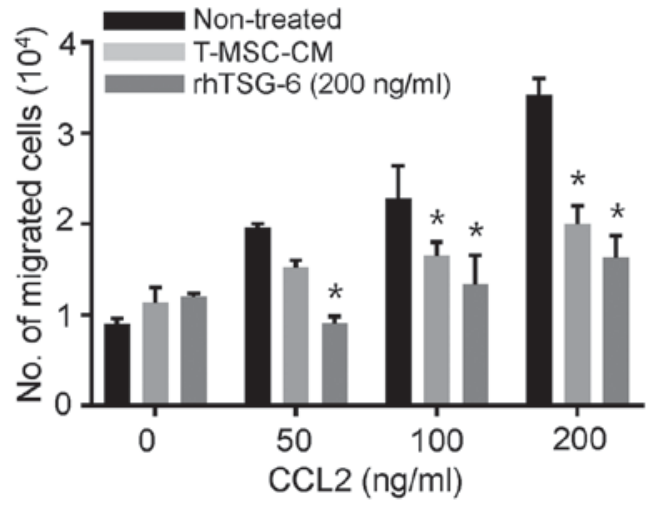

B

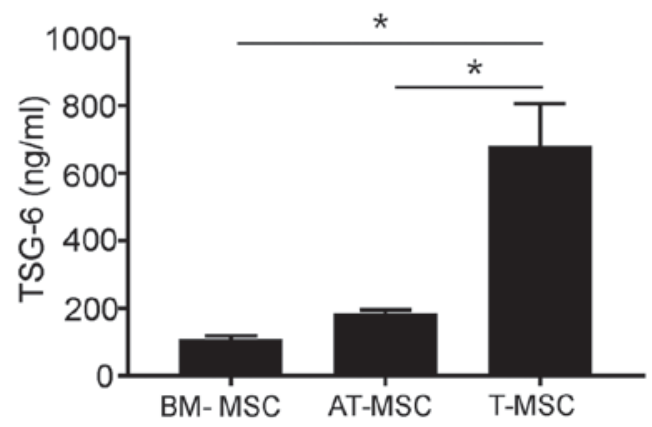

D

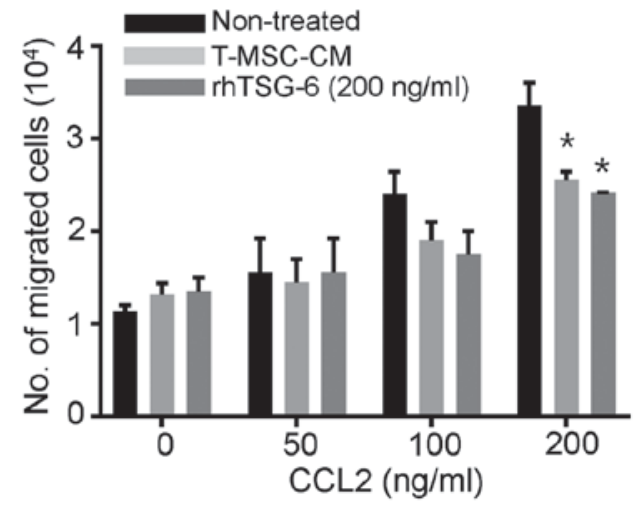

Figure 1. T-MSCs constitutively secrete TSG-6 and inhibit SPC and dLN cell chemotaxis in vitro. (A) Cell culture supernatants were collected and subjected to western blot analysis to detect the secreted form of TSG-6 in BM-MSCs, AT-MSCs and T-MSCs. DMEM media alone was loaded as the negative control. Cell lysates were harvested and endogenous levels of $\beta$-actin were detected for normalization. The pixel densities of the TSG- 6 bands were divided by the pixel densities of the corresponding $\beta$-actin bands. AT-MSC and T-MSC produce significantly higher extent of TSG-6 in comparison with those of BM-MSC. Data are presented as the mean \pm SEM. "P<0.05 vs. BM-MSC group). (B) TSG-6 levels in cell supernatants from BM-MSCs, AT-MSCs, and T-MSCs were measured by ELISA. Data are presented as the mean \pm SEM $\left({ }^{*} \mathrm{P}<0.05\right)$. The migration of mouse SPCs (C) and dLN cells (D) in response to CCL2 is shown using the Transwell migration assay. Data are presented as the mean \pm SEM. ${ }^{*} \mathrm{P}<0.05$ vs. Non-treated group. SEM, standard error of the mean; DMEM, Dulbecco's modified Eagle's medium; BM-MSC, bone marrow-derived mesenchymal stem cell; AT-MSC, adipose tissue-derived mesenchymal stem cell; T-MSC, tonsil-derived mesenchymal stem cell; TSG-6, tumor necrosis factor stimulated gene-6; T-MSC-CM, tonsil-derived mesenchymal stem cell conditioned medium; rhTSG-6, recombinant human tumor necrosis factor stimulated gene-6; SPC, spleen cell; dLN, draining lymph node; CCL2, chemokine (C-C motif) ligand 2.

T-MSC-CM attenuates the clinical manifestations of GVHD. A mouse model of GVHD was generated, as shown in Fig. 2A. GVHD was induced in mice conditioned with BU and CY prior to the injection of allogenic BMCs and SPCs. Despite the severe mortality of GVHD mice observed 7 days after cell transplantation, the addition of either T-MSC-CM or rhTSG- 6 prolonged survival (Fig. 2B). Rapid and severe weight loss was also observed in these mice within 7 days of BMC/SPC transplantation, which correlated with the mortality rates observed during that period. However, the administration of either T-MSC-CM or rhTSG-6 resulted in significantly lesser weight loss and faster recovery rates in comparison with GVHD mice (Fig. 2C).

T-MSC-CM or rhTSG-6 attenuates GVHD response. Major target organs of GVHD, including the skin, liver, small intestine, and large intestine, showed clear histopathological evidence of GVHD on day 7 (Fig. 3A) that was more severe than on day 21 (Fig. 3B). Skin samples exhibited a clear disruption of the epidermis and thinning of the dermis on day 7 post-transplantation, and liver samples showed extramedullary hematopoiesis and inflammatory infiltrates in the portal triad. Mucosal crypts in the small and large intestines were severely disrupted, exhibiting hyperplasia and hyperchromatic nuclei, with numerous cells displaying apoptotic characteristics. The total scores for GVHD severity are shown in Fig. 3C. This score was highest on day 21 in the GVHD group, but T-MSC-CM and rhTSG-6 improved this score by over 20 points.

T-MSC-CM and rhTSG-6 downregulate lymphocyte gene expression in GVHD-targeted organs. Because we observed significant inhibition of immune cell chemotaxis in the presence of T-MSC-CM, we speculated that the differential recruitment and expansion of lymphocytes, such as $\mathrm{T}$ or B cells, occurred in each experimental mouse group. To test our theory, we compared the expression of CD4 and CD19 in the liver, small intestine, and large intestine (Fig. 4). Our results revealed that GVHD greatly induced the expression of CD4 in all organs, whereas similar to the effects of rhTSG-6, T-MSC-CM significantly downregulated CD4 expression. The highest levels of CD19 expression were observed in the large 
A

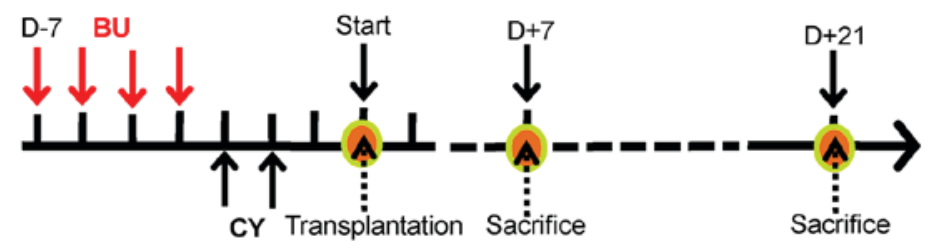

B

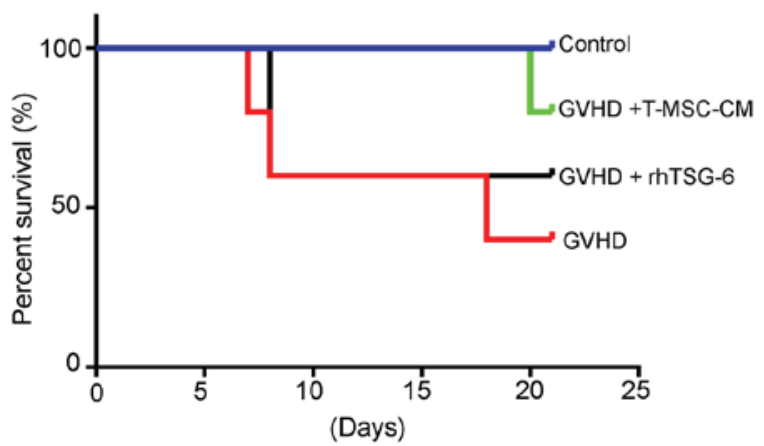

C

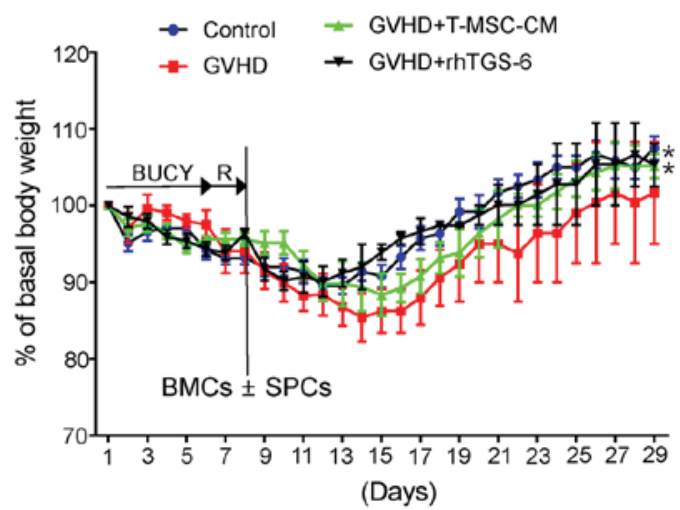

Figure 2. T-MSC-CM or rhTSG-6 reduces GVHD severity in mice. (A) Female BALB/c recipient mice received BU (20 mg/kg/day) for 4 days, followed by CY $(100 \mathrm{mg} / \mathrm{kg} /$ day $)$ for 2 days. All recipients received one day of rest before experimental transplantation. The mice were categorized into four groups: Transplantation of BMCs only (control); transplantation of BMCs plus SPCs (GVHD); BMCs plus SPCs with T-MSC-CM (GVHD-T-MSC-CM); and BMCs plus SPCs with rhTSG-6 (GVHD-rhTSG-6). After transplantation, the mice were monitored daily for 3 weeks. (B) A survival rate analysis of the different treatment groups ( $\mathrm{n}=8$ for each group) was performed using Kaplan-Meier estimates. (C) The total body weight of experimental BALB/C recipients was monitored over the study duration. Basal body weight was determined as weight at start. T-MSC-CM or rhTSG-6 treated mice showed significant recovery in comparison with GVHD group. Data are presented as the mean \pm standard error of the mean. " $\mathrm{P}<0.05$ vs. GVHD group. D, day; BU, busulfan; CY, cyclophosphamide; GVHD, graft-vs.-host disease; T-MSC-CM, tonsil-derived mesenchymal stem cell conditioned medium; rhTSG-6, recombinant human tumor necrosis factor stimulated gene- 6; BUCY, busulfan cyclophosphamide; R, recovery; BMCs, bone marrow cells; SPCs, spleen cells.

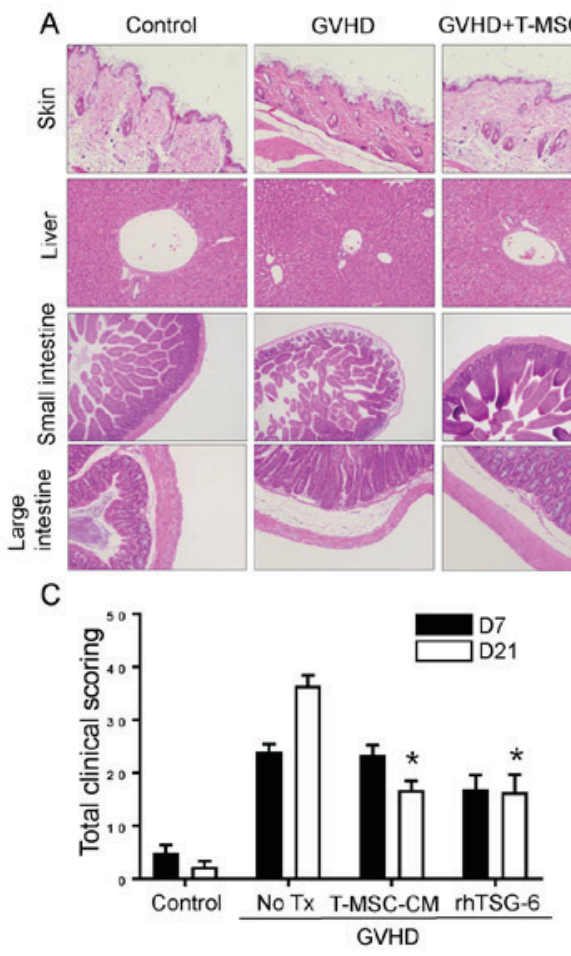

Figure 3. T-MSC-CM or rhTSG-6 attenuate the GVHD response. (A) Histological tissue sections of the skin, liver, small intestine, and large intestine were collected from experimental mice on day 7 post-transplantation (original magnification, x100 for liver and x 200 for skin, small intestine and large intestine). (B) Histological tissue sections of the skin, liver, small intestine, and large intestine were obtained from experimental mice on day 21 (original magnification, x100 for liver and x200 for skin, small intestine, and large intestine). (C) Slides of the skin, liver, small intestine, and large intestine obtained from experimental mice on days 7 and 21 post-transplantation were stained with hematoxylin and eosin and scored for GVHD severity according to standard criteria and added to determine the total score. Either T-MSC-CM and rhTSG-6 significantly reduce the scoring on post 21 day from GVHD induction. Data are presented as the mean \pm standard error of the mean. * $\mathrm{P}<0.05$ vs. GVHD group ( $\mathrm{n}=8$ ). GVHD, graft-vs.-host disease; T-MSC-CM, tonsil-derived mesenchymal stem cell conditioned medium; rhTSG-6, recombinant human tumor necrosis factor stimulated gene-6; No Tx, no treatment. 

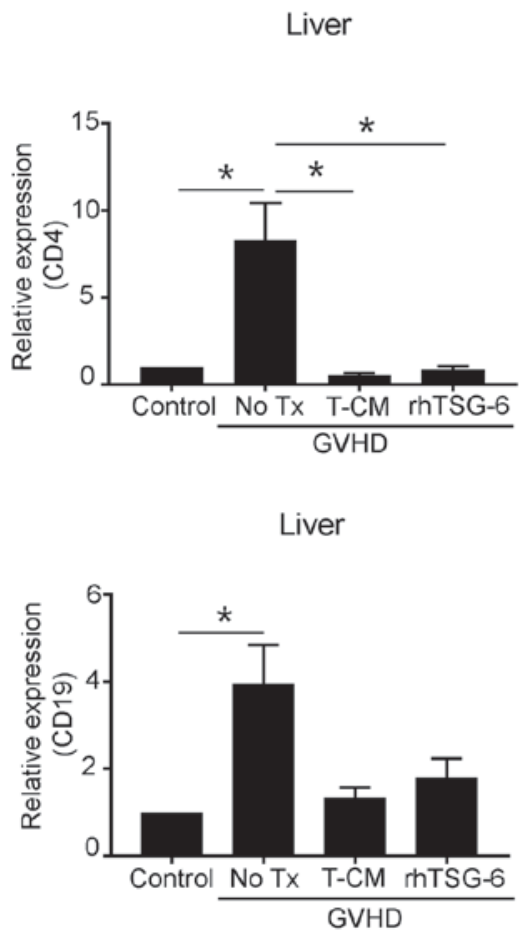
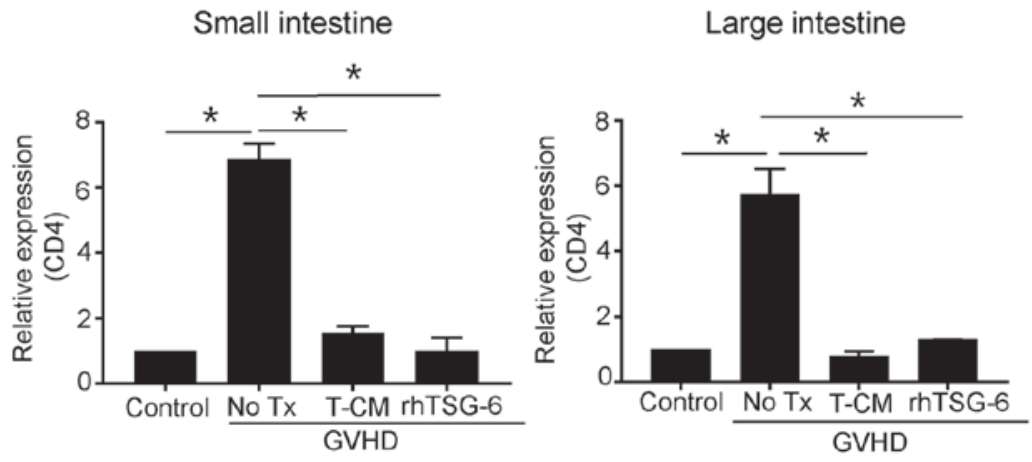

Small intestine

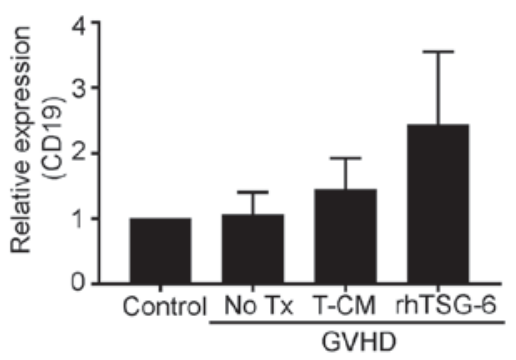

Figure 4. T-MSC-CM or rhTSG-6 downregulates lymphocyte gene expression in GVHD-targeted organs. Tissue samples from control, GVHD, GVHD-T-MSC-CM and GVHD-rhTSG-6 mice were collected on day 21 post-transplantation, and CD4 and CD19 gene expression were analyzed by reverse transcription-quantitative polymerase chain reaction. Data are presented as the mean \pm standard error of the mean ("P $<0.05$ as indicated). CD4, cluster of differentiation 4; No Tx, No treatment; T-CM, tonsil-derived mesenchymal stem cell conditioned medium; rhTSG-6, recombinant human tumor necrosis factor stimulated gene-6; GVHD, graft-vs.-host disease; CD19, cluster of differentiation 19.

intestine in GVHD mice, but these levels were reduced by T-MSC-CM. The liver also exhibited increased CD19 expression in GVHD mice that was inhibited by T-MSC-CM.

\section{Discussion}

In this study, we demonstrate that T-MSC-CM attenuates aGVHD responses in a mouse model of the disease. BALB/C recipient mice that were preconditioned by the administration of BU and CY were subsequently transplanted with BMCs and SPCs to induce GVHD. In this model, the administration of T-MSC-CM effectively prolonged survival, promoted a rapid recovery from weight loss and improved histological pathogenesis. Furthermore, lymphocyte expression levels of CD4 and CD19 were downregulated in GVHD mice injected with T-MSC-CM. Furthermore, the observed regulation of lymphocyte gene expression was supported by in vitro data, indicating that T-MSC-CM significantly inhibits the migration of diverse cell populations including immune cells.

The use of MSCs is a promising strategy for the treatment of aGVHD. A key advantage of MSCs is that histocompatibility matching is not required to achieve therapeutic effects. MSCs do not express human leukocyte antigen class II histocompatibility antigens or the CD40, CD80, or CD86 accessory molecules that are required for immune cell activation. An important biological property of MSCs is that their chemotactic responses to inflammatory factors are generally restricted to the migration of neutrophils and other immune-responsive cells. Once at the site of injury or inflammation, it is thought that MSCs modulate immune and inflammatory reactions at the microenvironmental level and stimulate tissue repair of affected organs (7). Several organs are targets in GVHD (skin, liver, and GI tract); thus, MSC therapy might depend on the number of infused cells that successfully traffic to various sites of tissue damage. However, the optimal number of cells and the number of administrations might be difficult to determine. Because more than one tissue is often damaged, the migration of cells to various tissues might not be uniform, leading to inefficient tissue repair. In fact, the use of an increased number of cells did not augment the therapeutic effects of BM-MSCs in GVHD (24). Regarding the route of cellular therapy, the direct, regional administration of cells to target organs in steroid-refractory GVHD was not as effective as their systemic injection (25). Thus, we assume that the therapeutic effects of MSCs in GVHD might be primarily attributed to the secretion of immunomodulatory factors following systemic infusion.

Previously, we reported that T-MSCs could serve as a cellular treatment for inflammatory conditions and the regeneration of damaged tissue via the abundant secretion of immunomodulatory proteins. Specifically, in this study, we found that T-MSCs produce high levels of TSG-6, a protein that inhibits immune cell chemotaxis, by directly binding to several chemokines. Of noted, we had difficulties in finding secretory loading protein that produced with similar extent from AT-MSCs, BM-MSCs, T-MSCs. Thus, we used $\beta$-actin as loading control protein in performing western blot. We believe $\beta$-actin can be alternative loading control protein because we cultured same numbers of cells for harvesting CM (AT-MSC, BM-MSC, and T-MSC) and used same concentration of protein for $\beta$-actin western blot. Due to its ability to inactivate chemokines, TSG-6 is assumed 
to play an inhibitory role in the development of GVHD by blocking donor cell migration into target organs that secrete a number of chemokines. When we tested whether rhTSG- 6 or T-MSC-CM could affect the migration of an immune cell population primarily composed of lymphocytes, both rhTSG-6 and T-MSC-CM significantly inhibited the chemotactic migration of responder cells. This finding supports the use of T-MSC-CM to treat GVHD, considering that the trafficking of donor $\mathrm{T}$ cells is critical for GVHD development. In fact, blocking the migration of donor $\mathrm{T}$ cells effectively attenuated aGVHD and preserved graft-vs.-leukemia activity in a tumor-bearing GVHD mouse model (18). In this prior study, blocking the chemotactic migration of donor $\mathrm{T}$ cells to GVHD-damaged organs efficiently enhanced their anti-tumor effects in a leukemic mouse model. In the current study, T-MSC-CM appeared to cause a similar inhibition of chemotaxis, possibly via the involvement of TSG-6.

Donor $\mathrm{T}$ cells were shown to partition to lymphoid tissues within h of a BMT. In the 2-3 days following transfer, allogeneic T cells expanded within lymphoid tissues. Between days 3 and 21, the number of allogeneic $T$ cells increased in GVHD-targeted organs, including the GI tract, liver, lung, and skin (26). This finding is consistent with our in vivo data, showing increased mortality 7 days after cell transplantation and more severe GVHD responses on day 21 post-transplantation compared with responses on day 7. Both rhTSG-6 and T-MSC-CM significantly recovered weight loss in GVHD mice, but T-MSC-CM showed higher therapeutic effect on survival. We assume survival might be affected by more complicated factors that are not restricted to weight recovery. For example, a mouse in mild weight loss with severely damaged target organ died earlier rather than mouse showed rapid weight loss with lesser extent of damages on target organs.

Further, our qPCR results support the critical pathophysiological sequence of events, as the expression levels of CD4 and CD19, surface antigens on T and B cells, respectively, were significantly increased on day 21 post-transplantation. Likewise, the downregulation of CD4 and CD19 expression by T-MSC-CM and rhTSG-6 suggests that immune cell migration and expansion were abrogated in GVHD organs. When considering that T-MSC-CM contains various immunomodulatory factors in addition to TSG-6, the effects of T-MSC-CM shown in this study might not be exclusive to TSG-6. For example, PD-L1 (27), IL-35 (12), and IL-1ra (13), which were previously reported to be anti-inflammatory proteins acting on effector $\mathrm{T}$ cell, $\mathrm{B}$ cells, or fibrosis, may also exert anti-inflammatory functions in GVHD. However, we believe that TSG-6 in T-MSC-CM may critically inhibit donor cell migration into GVHD-targeted organs rather than other factors in T-MSC-CM. The specific outcome of TSG- inhibition or the dual treatment of T-MSC-CM with rhTSG-6 or other molecules (e.g., PD-L1, IL-35 and IL-1ra) should be handled within our next expanded GVHD research.

In summary, we demonstrate that T-MSCs effectively ameliorate GVHD via secretory factors containing TSG-6. These findings suggest that T-MSC-CM could be a promising cellular agent for the treatment of transplant rejection.

\section{Acknowledgements}

Not applicable.

\section{Funding}

The present study was supported by a National Research Foundation of Korea (NRF) grant funded by the Korean government (grant no. NRF-2017R1E1A1A01073021). In addition, the present study was supported by the RP-grant 2018 of Ewha Womans University.

\section{Availability of data and materials}

The dataset used and/or analyzed during the current study are available from the corresponding author on reasonable request.

\section{Author's contribution}

$\mathrm{K}$-AC performed experiments and wrote the manuscript. Y-HK, MP and HJK also performed experiments and analyzed data. S-YW and J-WP also analyzed data and assisted in writing the manuscript. K-HR designed the experiments and assisted in writing the manuscript.

\section{Ethics approval and consent to participate}

All procedures were approved by the College of Medicine, Ewha Womans University Animal Care and Use Committee (approval no. ESM18-0403).

\section{Patient consent for publication}

Not applicable.

\section{Competing interests}

The authors declare that they have no competing interests.

\section{References}

1. Ferrara JL, Levine JE, Reddy P and Holler E: Graft-versus-host disease. Lancet 373: 1550-1561, 2009.

2. Bacigalupo A: Management of acute graft-versus-host disease. Br J Haematol 137: 87-98, 2007.

3. MacMillan ML, Weisdorf DJ, Wagner JE, DeFor TE, Burns LJ, Ramsay NK, Davies SM and Blazar BR: Response of 443 patients to steroids as primary therapy for acute graft-versus-host disease: Comparison of grading systems. Biol Blood Marrow Transplant 8: 387-394, 2002.

4. Deeg HJ: How I treat refractory acute GVHD. Blood 109: 4119-4126, 2007.

5. Perez L, Anasetti C and Pidala J: Have we improved in preventing and treating acute graft-versus-host disease? Curr Opin Hematol 18: 408-413, 2011.

6. Garnett C, Apperley JF and Pavlů J: Treatment and management of graft-versus-host disease: Improving response and survival. Ther Adv Hematol 4: 366-378, 2013

7. Wang Y, Chen X, Cao W and Shi Y: Plasticity of mesenchymal stem cells in immunomodulation: Pathological and therapeutic implications. Nat Immunol 15: 1009-1016, 2014.

8. Fajardo-Orduña GR, Mayani H and Montesinos JJ: Hematopoietic support capacity of mesenchymal stem cells: Biology and clinical potential. Arch Med Res 46: 589-596, 2015.

9. Dotoli GM, De Santis GC, Orellana MD, de Lima Prata K, Caruso SR, Fernandes TR, Rensi Colturato VA, Kondo AT, Hamerschlak N, Simões BP and Covas DT: Mesenchymal stromal cell infusion to treat steroid-refractory acute GvHD III/IV after hematopoietic stem cell transplantation. Bone Marrow Transplant 52: 859-862, 2017. 
10. Muroi K, Miyamura K, Okada M, Yamashita T, Murata M, Ishikawa T, Uike N, Hidaka M, Kobayashi R, Imamura M, et al: Bone marrow-derived mesenchymal stem cells (JR-031) for steroid-refractory grade III or IV acute graft-versus-host disease: A phase II/III study. Int J Hematol 103: 243-250, 2016.

11. Cho KA, Park M, Kim YH, Ryu KH and Woo SY: Mesenchymal stem cells inhibit RANK-RANKL interactions between osteoclasts and Th17 cells via osteoprotegerin activity. Oncotarget 8: 83419-83431, 2017.

12. Cho KA, Lee JK, Kim YH, Park M, Woo SY and Ryu KH: Mesenchymal stem cells ameliorate B-cell-mediated immune responses and increase IL-10-expressing regulatory B cells in an EBI3-dependent manner. Cell Mol Immunol: Jan 2, 2017 (Epub ahead of print)

13. Cho KA, Park M, Kim YH, Woo SY and Ryu KH: Conditioned media from human palatine tonsil mesenchymal stem cells regulates the interaction between myotubes and fibroblasts by IL-1Ra activity. J Cell Mol Med 21: 130-141, 2017.

14. Ryu KH, Cho KA, Park HS, Kim JY, Woo SY, Jo I, Choi YH, Park YM, Jung SC, Chung SM, et al: Tonsil-derived mesenchymal stromal cells: Evaluation of biologic, immunologic and genetic factors for successful banking. Cytotherapy 14: 1193-1202, 2012

15. Cantinieaux D, Quertainmont R, Blacher S, Rossi L, Wanet T, Noël A, Brook G, Schoenen J and Franzen R: Conditioned medium from bone marrow-derived mesenchymal stem cells improves recovery after spinal cord injury in rats: An original strategy to avoid cell transplantation. PLoS One 8: e69515, 2013

16. Gehmert S, Wenzel C, Loibl M, Brockhoff G, Huber M, Krutsch W, Nerlich M, Gosau M, Klein S, Schreml S, et al: Adipose tissue-derived stem cell secreted IGF-1 protects myoblasts from the negative effect of myostatin. Biomed Res Int 2014: 129048, 2014

17. Sadeghi B, Aghdami N, Hassan Z, Forouzanfar M, Rozell B Abedi-Valugerdi M and Hassan M: GVHD after chemotherapy conditioning in allogeneic transplanted mice. Bone Marrow Transplant 42: 807-818, 2008.

18. Cho KA, Woo SY, Park YS, Park MH and Ryu KH: Macrophage inflammatory protein-2 (MIP-2)/CXCR2 blockade attenuates acute graft-versus-host disease while preserving graft-versus-leukemia activity. Biochem Biophys Res Commun 426: 558-564, 2012.
19. Joo SY, Cho KA, Jung YJ, Kim HS, Park SY, Choi YB, Hong KM, Woo SY, Seoh JY and Ryu KH: Bioimaging for the monitoring of the in vivo distribution of infused mesenchymal stem cells in a mouse model of the graft-versus-host reaction. Cell Biol Int 35: 417-421, 2011.

20. Joo SY, Cho KA, Jung YJ, Kim HS, Park SY, Choi YB, Hong KM, Woo SY, Seoh JY, Cho SJ and Ryu KH: Mesenchymal stromal cells inhibit graft-versus-host disease of mice in a dose-dependent manner. Cy totherapy 12: 361-370, 2010.

21. Livak KJ and Schmittgen TD: Analysis of relative gene expression data using real-time quantitative PCR and the 2(-Delta Delta C(T)) method. Methods 25: 402-408, 2001.

22. Dyer DP, Thomson JM, Hermant A, Jowitt TA, Handel TM, Proudfoot AE, Day AJ and Milner CM: TSG-6 inhibits neutrophil migration via direct interaction with the chemokine CXCL8. J Immunol 192: 2177-2185, 2014.

23. Dyer DP, Salanga CL, Johns SC, Valdambrini E, Fuster MM, Milner CM, Day AJ and Handel TM: The anti-inflammatory protein TSG-6 regulates chemokine function by inhibiting chemokine/glycosaminoglycan interactions. J Biol Chem 291: 12627-12640, 2016.

24. Kebriaei P, Isola L, Bahceci E, Holland K, Rowley S, McGuirk J, Devetten M, Jansen J, Herzig R, Schuster M, et al: Adult human mesenchymal stem cells added to corticosteroid therapy for the treatment of acute graft-versus-host disease. Biol Blood Marrow Transplant 15: 804-811, 2009.

25. Arima N, Nakamura F, Fukunaga A, Hirata H, Machida H, Kouno S and Ohgushi H: Single intra-arterial injection of mesenchymal stromal cells for treatment of steroid-refractory acute graft-versus-host disease: A pilot study. Cytotherapy 12: 265-268, 2010.

26. Wysocki CA, Panoskaltsis-Mortari A, Blazar BR and Serody JS: Leukocyte migration and graft-versus-host disease. Blood 105: 4191-4199, 2005

27. Kim JY, Park M, Kim YH, Ryu KH, Lee KH, Cho KA and Woo SY: Tonsil-derived mesenchymal stem cells (T-MSCs) prevent Th17-mediated autoimmune response via regulation of the programmed death-1/programmed death ligand-1 (PD-1/PD-L1) pathway. J Tissue Eng Regen Med 12: e1022-e1033, 2018. 